\title{
Rival perspectives in health technology assessment and other economic evaluations for investing in global and national
}

\section{health. Who decides? Who pays? [version 1; peer review: 1}

\section{approved, 2 approved with reservations]}

\author{
Anthony Culyer(D1,2, Kalipso Chalkidou2,3, Yot Teerawattananon4, \\ Benjarin Santatiwongchai ${ }^{4}$ \\ ${ }^{1}$ Department of Economics \& Related Studies, University of York, UK, York, YO10 5DD, UK \\ ${ }^{2}$ Global Health and Development Group, Institute of Global Health Innovation, Imperial College London, London, SW7 2AZ, UK \\ ${ }^{3}$ Center for Global Development, Westminster Impact Hub, London, SW1Y 4TE, UK \\ ${ }^{4}$ Department of Health, Ministry of Public Health, Thailand, Nonthaburi, 11000, Thailand
}

V1 First published: 17 Jan 2018, 7:72

https://doi.org/10.12688/f1000research.13284.1

Latest published: 17 Jan 2018, 7:72

https://doi.org/10.12688/f1000research.13284.1

\section{Abstract}

There seems to be a general agreement amongst practitioners of economic evaluations, including Health Technology Assessment, that the explicit statement of a perspective is a necessary element in designing and reporting research. Moreover, there seems also to be a general presumption that the ideal perspective is "societal". In this paper we endorse the first principle but dissent from the second. A review of recommended perspectives is presented. The societal perspective is frequently not the one recommended. The societal perspective is shown to be less comprehensive than is commonly supposed, is inappropriate in many contexts and, in any case, is in general not a perspective to be determined independently of the context of a decision problem. Moreover, the selection of a perspective, societal or otherwise, is not the prerogative of analysts.

\section{Keywords}

HTA, CEA, perspective, stakeholder, social value judgments, reference case, patients' preferences.

\section{Open Peer Review}

$\begin{array}{lccc}\text { Approval Status } & ? \checkmark ? \\ & 1 & 2 & 3 \\ \text { version 1 } & ? & \checkmark & ? \\ \text { 17 Jan 2018 } & \text { view } & \text { view } & \text { view }\end{array}$

1. David Tordrup, Triangulate Health Ltd, London, UK University of Utrecht, Utrecht, The Netherlands

2. Alexander Winch $1 \mathrm{D}$, University of the Witwatersrand, Johannesburg, South Africa

3. Carol E. Levin ID, University of Washington, Seattle, USA

Any reports and responses or comments on the article can be found at the end of the article. 
Corresponding author: Anthony Culyer (tony.culyer@york.ac.uk)

Author roles: Culyer A: Conceptualization, Writing - Original Draft Preparation; Chalkidou K: Conceptualization, Project Administration, Writing - Review \& Editing; Teerawattananon Y: Project Administration, Writing - Review \& Editing; Santatiwongchai B: Writing Review \& Editing

Competing interests: No competing interests were disclosed.

Grant information: This work was supported by a grant from the Bill and Melinda Gates Foundation [OPP1134345] and the UK's Department for International Development. Yot Teerawattananon holds the Thailand Research Fund Senior Research Scholar grant for Health Technology Assessment for Supporting Universal Health Coverage (grant number RTA59800011).

The funders had no role in study design, data collection and analysis, decision to publish, or preparation of the manuscript.

Copyright: (c) 2018 Culyer A et al. This is an open access article distributed under the terms of the Creative Commons Attribution License, which permits unrestricted use, distribution, and reproduction in any medium, provided the original work is properly cited.

How to cite this article: Culyer A, Chalkidou K, Teerawattananon $Y$ and Santatiwongchai B. Rival perspectives in health technology assessment and other economic evaluations for investing in global and national health. Who decides? Who pays? [version 1; peer review: 1 approved, 2 approved with reservations] F1000Research 2018, 7:72 https://doi.org/10.12688/f1000research.13284.1

First published: 17 Jan 2018, 7:72 https://doi.org/10.12688/f1000research.13284.1 


\section{Introduction}

It seems a desirable thing to set international quality standards for conducting health technology assessments and other economic evaluations (EEs) of the impact and desirability of investments that promote health ${ }^{1}$. Such standards are, however, not easy to define, let alone to get agreement on ${ }^{2}$. What is easy is to fall into a trap of small-town thinking, by setting standards whose applicability is arbitrarily and quite needlessly restricted to: (a) particular cultural and political contexts, (b) within any such context, a set of choices bounded by further arbitrary value judgments, (c) a presumption that EE methods are designed for use only in the public sector - and, moreover, only when the object is "the public interest". Parochialism has two unattractive features. First, it denies the possible usefulness of EEs outside the public sector and to non-governmental agencies, like trade unions, for-profit companies and non-profit organisations like charities. Second, it imports value judgments to the effect that (a) EE can be conducted in a context-free way without attention being given to cultural and political values that may be particular to the application, which may not be readily transferable from jurisdiction to jurisdiction and (b) that the use of patients' (or the public's) preferences, in constructing outcome measure such as the quality-adjusted life year (QALY) or disability-adjusted life year (DALY) via estimated or expressed consumer values, rather than using, say, judgments made by parliamentary committees, expert groups or citizens' juries, provides an appropriate and relevant index of value. We seek to avoid such restraints by proposing that questions of perspective be determined in context, where the job of EE analysts is to help decision makers to articulate a perspective that is appropriate to the circumstances rather than to prescribe a perspective independently of its context of use (being "context free").

First, we review the recent history of "perspective".

\section{What is perspective and why does it matter?}

Guides to best practice in cost-effectiveness analysis (CEA) can be traced back at least as far as 1980, with the publication, at the initiative of Senate Committee on Labor and Human Resources and commissioned by the then Office of Technology Assessment (OTA), of The Implications of Cost-Effectiveness Analysis of Medical Technology ${ }^{3}$. Other significant landmarks appeared in 1994 by the Canadian Coordinating Office for Health Technology Assessment ${ }^{4}$, in 1996 by Gold et al. (the "Washington Panel") $)^{5}$, in 1997 by Drummond et al. ${ }^{6}$, in 2003 by the World Health Organization ${ }^{7}$, in 2004 by the National Institute for Clinical Excellence (NICE) ${ }^{8}$ and in 2016 by the second Panel $^{9}$ and the International Decision Support Initiative (IDSI) ${ }^{10}$.

The Washington Panel coined the term "Reference Case" to describe the characteristics of a high quality economic evaluation, thereby establishing both a lasting piece of terminology and launching a stream of literature (see original publication here: https://www.ncbi. nlm.nih.gov/pubmed/8861994 and over 170 references in PubMed: https://www.ncbi.nlm.nih.gov/pubmed?LinkName=pubmed_ pubmed_citedin\&from_uid=8861994). The Panel's work absorbed much of that by Drummond et $a l^{6}$. One of the characteristics of a high-quality EE was the explicit stating of a study's "perspective", described by the panel as "the study's point of view", which "determines which health outcomes and costs are relevant and plays a part as well in how they should be valued". We wholly agree with this principle and it seems to have formed a part of all subsequent reference cases, including one developed by the Drummond et al. team and us, through the Gates-sponsored iDSI Reference Case (http://www.idsihealth.org/resource-items/idsi-referencecase-for-economic-evaluation/). The case for it is made not merely on grounds of transparency, which matters because it enables other researchers to check that the scope and measures of costs and benefits were indeed fit for purpose, but also on grounds of accountability: what to include and how to measure it are essential political and social value judgments that ought to be made by duly accountable decision makers (not EE analysts!). A good study will be, therefore, explicit about its perspective (or perspectives, if more than one is to be explored).

The Washington Panel recognised that a perspective must reflect the purpose of a study. It is not always appreciated that the perspective will usually be context-dependent, with the context determining the appropriate perspective. For example, a study might appraise the costs and benefits of a workplace intervention to reduce back strain on workers through redesigned work stations from the points of view of managers on the one hand and trade unions on the other, with a view to anticipating opposition and identifying compensatory and regulatory measures for successful implementation. In such a case, there are three perspectives to be considered - those of management, workers and government. The perspectives to be used are defined by the purpose of the study which in turn is specified by its sponsor: in this example, management, workers or the government (or a consortium of all three).

\section{When it comes to perspectives, is broader better?}

The First Washington Panel's Reference Case recommended that a societal perspective be adopted, offering decision makers as wide as possible an understanding of the costs and consequences of alternative actions both in the health care sector and beyond it into the private health care sector and non-health sectors, public or private. Its reasoning is plain: only if the societal approach is adopted can decision makers be confronted with a full information set of the costs and consequences of alternative actions. Anything less comprehensive will necessarily be subject to omitted variable bias, probably of unknown sign and size, causing either over- or under-investment in both old and new technologies. It is hard to raise an objection of principle to this. Pragmatically, however, to seek inclusion of every consequence, no matter what the costs are of tracking it down and quantifying it, seems completely over the top: the limits of inclusion ought more rationally to be determined by a sensitivity analysis of whether inclusion or exclusion makes a difference of significant concern to any relevant outcome. At any rate, reasonably full information might be conceded as a desirable thing when the objective is to produce a decision that is in the public interest. What constitutes "reasonable" in any given context would necessarily be a matter for local judgment (decision makers and advisers). If the objective were less comprehensive than societal, however, to collect full information would be to collect much that was irrelevant to the context of that decision question. The argument that "more 
information is always better" is therefore not an argument for adopting the societal perspective; it is an argument for EEs that can reflect many perspectives, including the "societal" one, but only up to the point at the value of further information is judged to be less than the cost of collecting and incorporating it.

What "societal" means is, even in its most comprehensive version stated in the literature, commonly rather more restricted in terms of the nature of the consequences considered than may be thought. Compassionate externalities ${ }^{11-13}$ are usually not included. Stresses and welfare losses resulting from changes in delivery pathways, provider identity, loss of employment (temporary or permanent), limited involvement in or being informed about changes, confusion and misunderstanding of the purposes of changes - such effects characteristically accompany both investment decisions and changes in the ways in which decisions are made but none, however, feature in the scope of "societal" as commonly stated. "Societal" is thereby significantly short of being as comprehensive as may appear.

In any event, in practice, not all have followed the Panel's recommendation. Table 1 provides an illustrative variety of approaches. As would be expected, perspectives offered by an academic do not necessarily reflect the expressed views of public agencies. Developed countries like England, Australia and Canada rely on EE to inform allocation decisions and tend to be narrower in their scope of analysis whereas global Reference Cases tend to adopt a wider perspective. Some low-income and middle-income countries (LMICs), where out-of-pocket costs are significant select a societal perspective though very few have a track record of using EE systematically.

The limits of feasibility are much more tightly drawn in LMICs than in rich countries, where a closer approximation to "full information" may be both possible and desirable. In LMICs, what is possible is much more limited due to poor or absent data, lack of technical skills to conduct and use EEs, and lack of political understanding, leadership and support ${ }^{14,15}$. Table 2 shows that a healthcare payer's perspective is the most popular recommendation in the national guidelines of high-income countries where EE is commonly used to inform reimbursement of health services. Only a third of the guidelines from both high-income countries and LMICs recommend a societal perspective.

It would also be rash to suppose that what is regarded as the public interest is always and everywhere the same. Just such a belief underlies the World Health Organization (WHO) advocacy of DALYs as a kind of universal outcome metric with weights attached to dimensions of disability that are invariant with respect to culture and context. This is advanced as an advantage of DALYs over QALYs, since QALYs typically use customized country weights. A similar insensitivity can be seen in the WHO's abandoned attempts to recommend thresholds ${ }^{16}$. A threshold is an aggregate expression of collective willingness to pay for treatments in national health insurance. It represents a marginal preference for public expenditure on health care relative to the many other objects of public policy - poverty reduction, education and training, housing, law and order, civil and national defence, and so on. There can be no presumption that one size fits all, regardless of political stability, stage of economic development, culture, religion, or history. We should not encourage a similar insensitivity to enter an authoritative methodological guide to best practice in EE.

\section{Societal perspective: Whose values and whose judgments count?}

A problem with the use of a societal perspective is that it raises the possibility of reintroducing into health policy decisions many of the very biases that most health care systems have been designed to avoid. A critically important one concerns the implications of a perspective for the role of valuation and willingness to pay for health gains. It is part of the conventional wisdom of health economics that the health care market is characterised by more market failures - and serious ones at that - than almost any other market. Many of these impinge on the reasonableness of using individual willingness to pay as a priority-setting criterion and, more generally, of using the Pareto criterion (or the potential Pareto criterion) to identify socially beneficial changes ${ }^{17}$. These are standard aspects of the textbook treatments of heath economics: we merely list those that are of chief concern (Table 3). It is not satisfactory, as in Pauly (1995) $)^{18}$ and others who advocate the use of consumer preferences as a basis for public priority setting, to glide over these issues as if they were empirically or politically unimportant. The problems of reliance on consumer preferences for healthcare relate not only to measures of individual (as distinct from collective) willingness to pay but to all expressions of patient preferences. They raise the difficult question of the right balance to be struck between individual expressions of value and collective ones.

This is not the place to argue the cases for or against individual willingness to pay as a basis for making health investments or of locating the patient as a decision maker in a healthcare system. Our purpose is only to argue that a societal perspective need not necessarily require its source of value, whether or not monetised, to be individual patients. The welfare of patients remains a central concern but avoiding the distortions of the real world is necessary if patients are to be responsibly protected.

What, therefore, is the appropriate source for social valuations of the kind used in EE? And whose willingness to pay for the treatments offered in public insurance benefit packages of the sort aspired to, especially in LMICs, should be embodied in policy? We are not concerned here with individual choices of clinical providers and treatments, though the bulleted issues below do have implications for information provision, clinical audit and health care quality assurance. The questions for EE concern instead the value content of a set of key collective judgments that usually have to be made. The answer to all of the following will be shaped by the perspective:

- The choice of outcome measure (life-years? Lives? DALYs? QALYs? Market value of human capital generated, or commercial gain?).

- The construct validity of the possible measures, given the values and culture of country in question (which may 


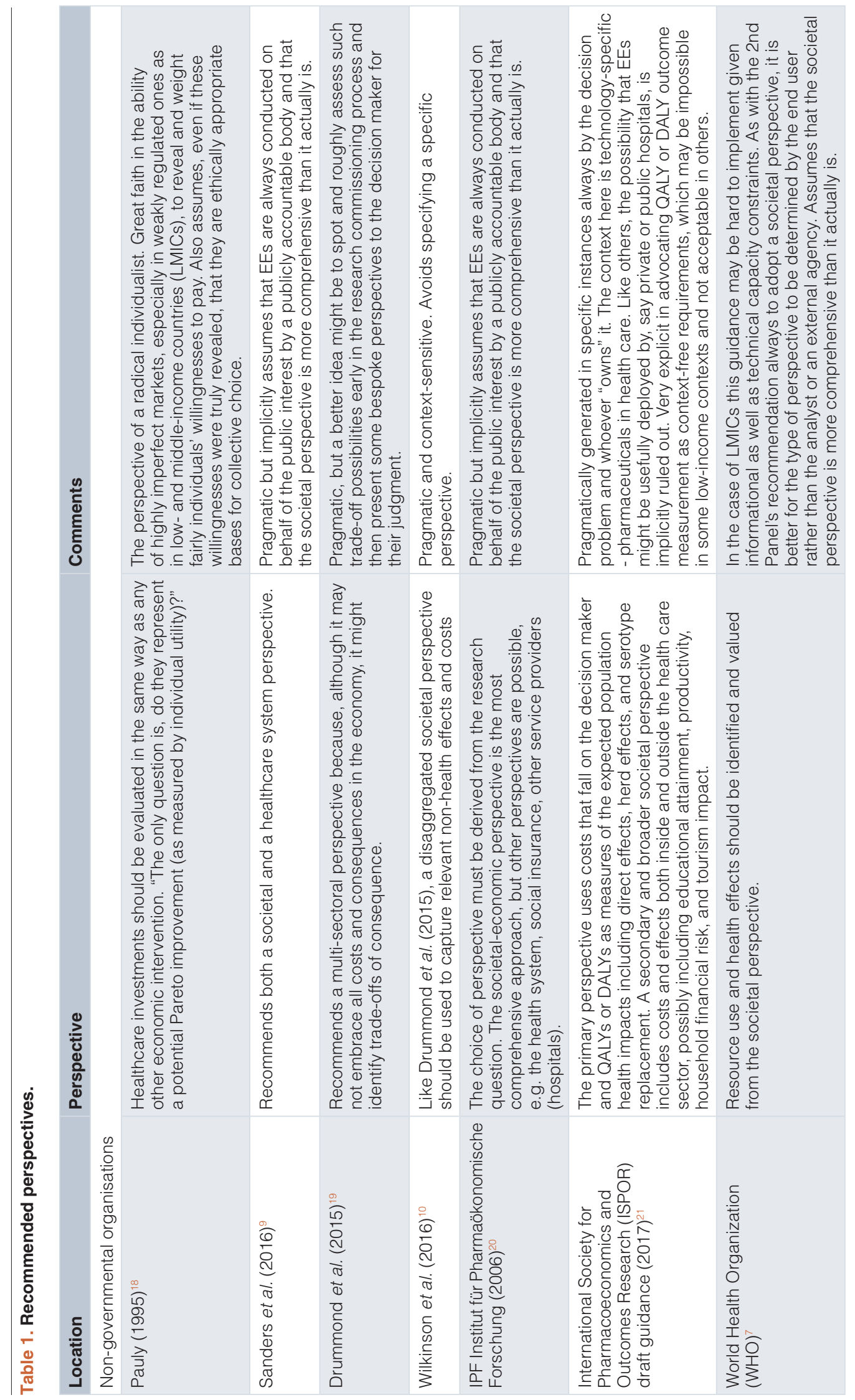




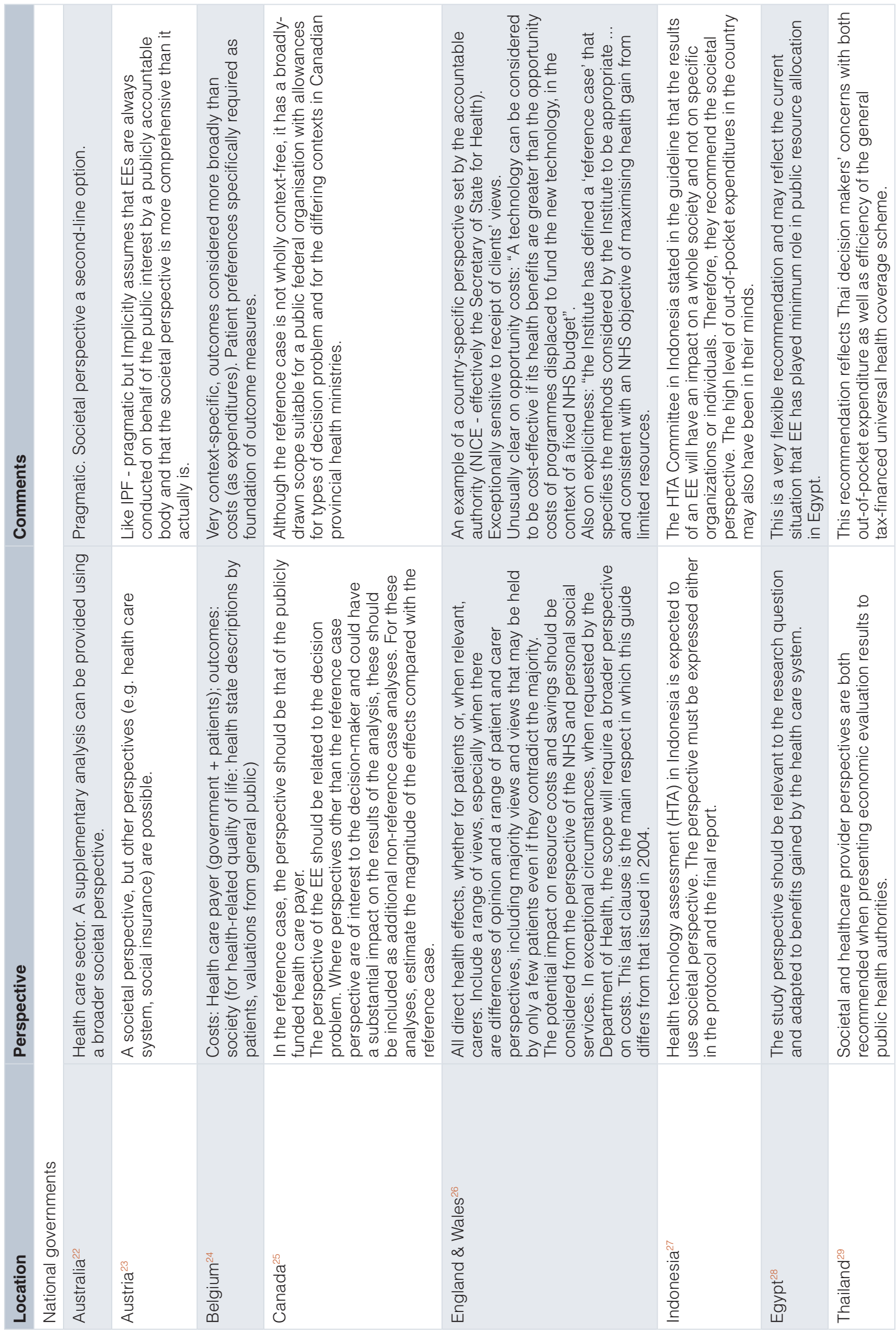


Table 2. Perspectives recommended by national methodological guidelines.

\begin{tabular}{|l|r|r|r|}
\hline Perspectives & $\begin{array}{c}\text { High-income } \\
\text { country guidelines }\end{array}$ & $\begin{array}{c}\text { Low- and middle-income } \\
\text { country guidelines }\end{array}$ & \multicolumn{1}{c|}{ Total } \\
\hline Payer & $14(47 \%)$ & $4(36 \%)$ & $18(44 \%)$ \\
\hline Health care sector & $6(20 \%)$ & $3(27 \%)$ & $9(22 \%)$ \\
\hline Societal & $10(33 \%)$ & $4(36 \%)$ & $14(34 \%)$ \\
\hline Total & $30(73 \%)$ & $11(27 \%)$ & $41(100 \%)$ \\
\hline
\end{tabular}

Source: GEAR's guideline comparison http://www. gear4health.com/gear/health-economicevaluation-guidelines

themselves be heterogeneous across regions/provinces). ("Does this measure correspond sufficiently to what we collectively understand to be the underlying concept (health)?")

- The impact of the country's budget for health care on the affordability of the various potential benefit packages (or extensions to them). ("What would a modest addition to or subtraction from the current level of public expenditure on health and healthcare imply for additional possible interventions, or for those most likely to be removed from the benefits package?")

- The relative value in real terms (opportunity cost) and at the margin of the various interventions. ("Do we need a money measure or is it sufficient to compare the impact of each on health?")

- The relative value in real terms (opportunity cost) and at the margin of extensions of entitlement. ("How do we trade-off the benefits of extending the benefits package to more people or specific groups (dwellers in remote places, workers, civil servants, the military...) against increasing the scope and size of the package for existing members of the scheme?")

- The relative value in real terms (opportunity cost) and at the margin of increased financial protection from the hazard of catastrophic (or significantly large) personal healthcare expenditure. ("Which population groups are most likely to benefit and what is a good measure of that benefit?")

- The monetary value of an increment in outcome required if an intervention is to be added to the benefit bundle or to displace existing benefits. ("What price adjustments must the manufacturer offer for this intervention to become cost-effective?")

The collective answers to these questions require no knowledge of individual willingnesses to pay for health care. Instead they require decisions taken at the societal level by decision makers accountable through some locally legitimate political process, advised where appropriate by expert opinion and supported by the best available evidence. Individual preferences/values might be considered relevant in selecting outcome measures, their dimensions (physical, mental functioning, etc.), the weights attached to various dimensions that enable them to be added up to create an index of outcome, and the weights (commonly unity) to attach to individuals when individual levels of health or disability are aggregated to community level. Decision makers need to exercise judgment regarding the extent to which individual members of a community are to be involved in these judgments. The societal judgments that are required are themselves choices that should be context-dependent and essentially locally accountable.

"Societal" does not necessarily imply "everyone". Decision makers may have their own views about whom to consult and when. They will have their own views as to what exposures to risk and distributions of cost and benefit are fair. They may have general across-the-board financial rules for public sector investments that stipulate discount rates and time horizons. They will address at a higher level what can be afforded for health care in the light of pressing needs for education, housing, poverty relief, law and order, etc. They will decide whether their constitutional preference is to narrow the idea of health to whatever impact health care provision has on it. They will decide whether to consider non-health consequences of health care investments.

\section{Who are the stakeholders? A tentative list}

Stakeholders all have perspectives, some of which may be shared, but most of which will need to be accommodated in some way in the methods of EE and the processes through which it is conducted. The extent to which individual ideas about perspective can be effectively suppressed in the interest of having a shared and publicly stated perspective has not yet, so far as we are aware, been explored empirically. EE analysts can give guidance. For example, a suggested list of possible stakeholders whose roles are for decision makers to consider (which has to be contextsensitive) might be as follows:

- Representative of the general (or relevant regional) public

- Patients with personal or vicarious experience of a relevant condition

- Family informal carers

- Relevant clinical specialists

- Clinical generalists 
Table 3. Problems with relying on patient preferences.

\section{Issue \\ Socio-economic gradient} linking health and wealth

Principal-agent discordances

Asymmetrical information

gnorant or prejudiced clinical judgments

Irrational behaviour

Externalities

Public goods

\section{Characteristics and consequences}

III-health and disability are inversely related to ability to pay (income or wealth). At all points on the gradient the better off have better general health and less disability. Willingness to pay is increased by ability to pay so those most in need of healthcare are least able to afford it. Consequently, willingness to pay as a criterion directs resources away from the neediest and will similarly direct collective investments in healthcare technologies 30,31

An agent acts on behalf of a principal and is supposed to serve the principal's interest. A physician's professional role can become compromised if their private financial interests clash with the patient's need for medication or with best-practice guidelines. This may be the case with fee-for service systems of physician remuneration, systems in which physicians dispense as well as prescribe, when there is no institutional support for producing and following authoritative clinical guidelines, and where patients' choices are influenced by biased advertising and inaccurate web-sourced information. These discordances may make patients' true preferences or willingnesses to pay impossible to detect reliably ${ }^{32}$.

A form of principal-agent corruption, in which a demand for ineffective care (such as needless office visits, prescription of ineffective medicines, use of ineffective or harmful surgical procedures) is manufactured by the physician. A consequence is that the value placed on healthcare is exaggerated and promoted by commercially supported patient advocacy groups ${ }^{33-35}$.

The most common form of asymmetry is that between physician and patient. Each has knowledge not possessed by the other. Patients are usually experts on their own fears and insecurities, financial and family circumstances, work-related and other social obligations. The professional is usually more expert in diagnosis and understanding treatment options and their health consequences. Problems arise when such knowledge is not communicated and shared - for example the patient's own expert knowledge of their personal and family circumstances is ignored, or the nurse's advice and the reasons for it are poorly given, or understood, or not followed (or all three).

Other asymmetries can arise, for example between general practitioners and specialists, clinical professionals and managers, though these have a lesser bearing on the revelation of patients' preferences and values.

Asymmetries raise questions about the adequacy of patient preferences, as expressed in their willingness to pay or through surveys, as representations of patients' true interests ${ }^{36,37}$.

Because the demand for healthcare is almost always evidenced through decisions taken by a clinician, poor training or failure to maintain professional standards can result in choices that are not representative of patients' values or best interests even when the other imperfections are absent. This is especially the case with so-called "complementary" medicine and, in LMICs with "traditional" medicine, little of which is evidence-informed, much of which is unhygienic, some of which is directly harmful, and all of which is conducted in a commercial way. A consequence is that expressed patient preferences need to be interrogated lest they are based on faulty or incorrect clinical understanding ${ }^{38}$.

The theory underlying the use of willingness to pay as a measure of patient benefit is built upon a set of assumptions about human choice behaviour, all of which have been exposed as false for at least some of the time. The most common (utilitarian) assumptions are: Completeness: either $A$ is preferred to $B$, or $B$ to $A$ or a person is indifferent between them; Transitivity: if $A$ is preferred or indifferent to $B$ and $B$ is preferred or indifferent to $\mathrm{C}$, then $\mathrm{A}$ is preferred or in-different to $\mathrm{C}$; Continuity: there is an indifference curve such that all points to its north-east are preferred to all points to its south-west; Convexity: the marginal rate of substitution is negative; Non-satiation: more is always preferred. If one or more of these axioms is violated the link between choice and welfare is broken and, indeed, the nature of "rationality" becomes ambiguous ${ }^{39}$.

In some circumstances patients are inherently incompetent in whole or part ${ }^{40}$, typically through illness or disability, youth or old age. In consequence, any value expressed may need careful interpretation.

An externality exists when the actions of one person have direct impact on the welfare of another. These may be physical, as when being immunised reduces the risk to others of falling ill (up to herd immunity), or psychic, as when the mere knowledge that poor people have medical coverage increases the welfare of people with sympathetic feelings. A consequence of such effects is that the benefit to the patient underestimates the social benefit (i.e. the sum of the private benefit to the patient and the external benefit to one or more others ${ }^{11,41}$.

This notion of publicness has nothing to do with the ownership of resources. It refers to the nature of benefit. A public good is one from one from which consumers cannot be excluded (whether or not they pay $)^{42}$. Classic examples are street lighting and national defence - whether you like it or not you receive the service. Public health measures often have this characteristic: clean water supply and proper waste disposal benefit all (including people downstream who are part of another community). The external benefit of immunisation is generally a public benefit. The theoretically correct way of estimating the social value of such interventions is by summing the value to all who benefit from each scale of operation or, for increases in such interventions, summing the willingness to pay each has for the addition. Apart from the impracticability, this approach is also subject to some of the other objections to the use of willingness to pay. Consequently, an overall judgment has to be made on behalf of the entire community in question 
- Professional leaders (early adopters)

- Politicians (including the opposition parties)

- Researchers (all relevant disciplines)

- Product manufacturers

- Health service providers

- Health service managers

- Aid givers and donor organisations

- Relevant NGOs and other sponsors

- Insurers (public and private)

- Educators

- Media communications specialists and journalists

- Parliamentarians

- The Judiciary

- Other interested parties

Selection of stakeholders and possible roles they might play in determining the perspective are matters on which EE analysts may advise, for example, by listing options, sharing experience elsewhere, indicating likely consequences, and building models.

\section{On the role of the analyst (and how we differ from others)}

Our recommendation is, then, that analysts should approach the issue of perspective at two levels of specificity: the universal or general (context-free) on the one hand, and the context-dependent on the other. A fundamental universal context-free principle is that the perspective always be stated, be consistently adhered to throughout an EE study and appear in reports of the study. Another is that the reasons for the perspective should be given, any departures from previous or common practice elsewhere noted and explained.

These two principles will also shape much of the process in conducting an EE; for example, identifying partners and stakeholders, the extent of consultation, the need for new context-specific research, time-lines and reporting accountability. Their roles will depend upon the context-specific perspective actually adopted. For example, most perspectives will doubtless need to include physicians and patients in consultation meetings, but the scope of the issues discussed will differ according to the specific perspectives: in some studies they might advise on the probable accuracy of the outcome measure and the likely cost consequences of adopting a new technology in the social care sector, in others, they may be concerned only with the outcome measure. The national payer may specify that no additional resources will be available, which will encourage a relatively narrow perspective. Alternatively, the national payer may want an EE performed in order to inform a review of public expenditure across all sectors, in which case a broader public-sector perspective is implied. A health advocacy group, by contrast, may wish a societal perspective to be adopted to establish a case for increasing the health budget. A public payer committed to health maximisation is likely to adopt a perspective that focuses narrowly on health as an outcome while being much broader in its consideration of cost-effective means of promoting health by embracing nutrition, public education, housing and other determinants not normally within the direct control of the Ministry of Health. In each case the perspective is determined by the interests of the client for the analysis. A donor committed to universal health care might adopt any of a number of positions regarding perspective, depending both on its fundamental strategic purposes (perhaps with a single disease perspective) and on the specific context of application (perhaps having specific regard to local capacities to manage complex delivery requirements).

\section{Conclusions}

Our conclusions can be simply stated:

- adopting a societal perspective is not a context-free requirement,

- being explicit about the perspective is a context-free requirement,

- choice of perspective is always and everywhere a matter for the study sponsors to determine together with any stakeholders they select,

- if the sponsors of a study intend it to inform policy decisions (public or otherwise) the decision makers' perspective should be adopted (along with others deemed to be relevant by the sponsors),

- the role of economists, epidemiologists and the like is to advise, assist, point out likely consequences, and to seek out, adduce, model and interpret evidence,

- the role of economists, epidemiologists and the like is not to engage in advocacy for specific context-dependent perspectives, societal or otherwise.

\section{Competing interests}

No competing interest were disclosed.

\section{Grant information}

This work was supported by a grant from the Bill and Melinda Gates Foundation [OPP1134345] and the UK's Department for International Development. Yot Teerawattananon holds the Thailand Research Fund Senior Research Scholar grant for Health Technology Assessment for Supporting Universal Health Coverage (grant number RTA59800011).

The funders had no role in study design, data collection and analysis, decision to publish, or preparation of the manuscript. 
1. Bell CM, Urbach DR, Ray JG, et al.: Bias in published cost effectiveness studies: systematic review. BMJ. 2006; 332(7543): 699-703. PubMed Abstract | Publisher Full Text | Free Full Text

2. Philips Z, Ginnelly L, Sculpher M, et al:: Review of guidelines for good practice in decision-analytic modelling in health technology assessment. Health Technol Assess. 2004; 8(36): iii-iv, ix-xi, 1-158. PubMed Abstract | Publisher Full Tex

3. Office of Technology Assessment: The Implications of Cost-Effectiveness Analysis of Medical Technology. Washington, DC: Congress of the United States; 1980.

Reference Source

4. Canadian Coordinating Office for Health Technology Assessment (CCOHTA): Guidelines for economic evaluation of pharmaceuticals. first edition ed. Ottawa: CCOHTA; 1994.

5. Gold MR, Siegel JE, Russell LB, et al:: Cost-effectiveness in health and medicine. New York: Oxford University Press, 1996.

Reference Source

6. Drummond MF, Stoddart GL, Torrance GW: Methods for the economic evaluation of health care programmes. Second edition ed. Oxford: Oxford University Press; 1997.

Reference Source

7. World Health Organization: Making choices in health: WHO guide to costeffectiveness analysis. Tan-Torres Edejer T, Baltussen R, Adam T, Hutubessy R, Acharya A, Evans DB, et al., editors. Switzerland, 2003. Reference Source

8. National Institute for Health and Clinical Excellence (NICE): Guide to the methods of technology appraisal. London: NICE, 2004 Reference Source

9. Sanders GD, Neumann PJ, Basu A, et al: Recommendations for Conduct, Methodological Practices, and Reporting of Cost-effectiveness Analyses: Second Panel on Cost-Effectiveness in Health and Medicine. JAMA. 2016; 316(10): 1093-103

PubMed Abstract | Publisher Full Text

10. Wilkinson $\mathrm{T}$, Sculpher MJ, Claxton K, et al:: The International Decision Support Initiative Reference Case for Economic Evaluation: An Aid to Thought. Value Health. 2016; 19(8): 921-8

PubMed Abstract | Publisher Full Text

11. Jacobsson F, Carstensen J, Borgquist L: Caring externalities in health economic evaluation: how are they related to severity of illness? Health Policy. 2005 73(2): 172-82

PubMed Abstract | Publisher Full Text

12. Culyer AJ: The Political Economy of Social Policy. Oxford: Martin Robertson; 1983.

13. Culyer AJ: The normative economics of health care finance and provision. Oxf Rev Econ Policy. 1989; 5(1): 34-58. Publisher Full Text

14. Yothasamut $\mathrm{J}$, Tantivess $\mathrm{S}$, Teerawattananon $\mathrm{Y}$ : Using economic evaluation in policy decision-making in Asian countries: mission impossible or mission probable? Value Health. 2009; 12(Suppl 3): S26-S30. PubMed Abstract | Publisher Full Text

15. Hipgrave DB, Alderman KB, Anderson I, et al.: Health sector priority setting at meso-level in lower and middle income countries: lessons learned, available options and suggested steps. Soc Sci Med. 2014; 102: 190-200. PubMed Abstract | Publisher Full Text

16. Bertram MY, Lauer JA, De Joncheere K, et al:: Cost-effectiveness thresholds: pros and cons. Bull World Health Organ. 2016; 94(12): 925-30. PubMed Abstract | Publisher Full Text | Free Full Text

17. Reinhardt UE: Abstracting from distributional effects, the policy is efficient In: Barer ML, Getzen TE, Stoddart GL, editors. Health, Health Care and Health Economics. New York: Wiley; 1998; 1-52.

18. Pauly MV: Valuing health benefits in monetary terms. In: Sloan F, editor. Valuing health care Costs, benefits and effectiveness of pharmaceutical ad other medical technologies. Cambridge: Cambrige University Press; 1995. Reference Source

19. Drummond MF, Sculpher M, Claxton K, et al:: Methods for the economic evaluation of health care programmes. Fourth edition ed. Oxford: Oxford University Press; 2015.

Reference Source
20. Walter E, Zehetmayr S: Guidelines zur gesundheitsökonomischen Evaluation Konsenspapier. Wien Med Wochenschr. 2006; 156(23-24): 628-32. Publisher Full Text

21. ISPOR RCT-CEA Taskforce. Unpublished report. 2017.

22. Department of Health of Australian Government: Guidelines for preparing a submission to the Pharmaceutical Benefits Advisory Committee (version 5.0). 2016.

Reference Source

23. Walter E, Zehetmayr S: Guidelines on health economic evaluation. 2006 Reference Source

24. Cleemput I, Neyt M, Van de Sande S, et al.: Belgian guidelines for economic evaluations and budget impact analyses: second edition. Brussels: Belgian Health Care Knowledge Centre (KCE), Contract No.: D/2012/10.273/54. 2012 Reference Source

25. Guidelines for the economic evaluation of health technologies: Canada. 4th ed Ottawa: CADTH, 2017. Reference Source

26. National Institute for Health and Care Excellence: Guide to the Methods of Technology Appraisal 2013 [Internet]. 2013.

PubMed Abstract

27. Ministry of Health: Health technology assessment guidelines. 2017.

28. Ministry of Health and Population: Guidelines for Reporting Pharmacoeconomic Evaluations. Reference Source

29. Tanvejsilp P, Ngorsuraches S: Defining the scope of health technology assessment and types of health economic evaluation. J Med Assoc Thai. 2014; 97(Suppl 5): S10-6.

PubMed Abstract

30. Marmot MG, Smith GD, Stansfeld S, et al.: Health inequalities among British civil servants: the Whitehall II study. Lancet. 1991; 337(8754): 1387-93. PubMed Abstract | Publisher Full Text

31. Deaton A: Policy Implications of the gradient of health and wealth. Health Aff (Millwood). 2002; 21(2): 13-30. PubMed Abstract | Publisher Full Text

32. Arow KJ: The economics of agency. In: Pratt J, Zeckhauser R, editors. Principals and Agents. Cambridge, MA: Harvard Business School; 1985; 37-51. Reference Source

33. Dranove D, Wehner P: Physician-induced demand for childbirths. $J$ Health Econ 1994; 13(1): 61-73. PubMed Abstract | Publisher Full Text

34. Scott $A$, Shiell A: Do fee descriptors influence treatment choices in general practice? A multilevel discrete choice model. J Health Econ. 1997; 3: 303-21.

35. Yip WC: Physician response to Medicare fee reductions: changes in the volume of coronary artery bypass graft (CABG) surgeries in the Medicare and private sectors. J Health Econ. 1998; 17(6): 675-99. PubMed Abstract | Publisher Full Text

36. Rochaix L: Information asymmetry and search in the market for physicians services. J Health Econ. 1989; 8(1): 53-84. PubMed Abstract | Publisher Full Text

37. Reinhardt UE: Can efficiency in health care be left to the market? $J$ Health Polit Policy Law. 2001; 26(5): 967-92. PubMed Abstract | Publisher Full Tex

38. Leonard K, Mliga G, Mariam DH: Bypassing health centres in Tanzania: revealed preferences for quality. J Afr Econ. 2002; 11(4): 441-71. Publisher Full Text

39. Rieskamp J, Busemeyer JR, Mellers BA: Extending the bounds of rationality: evidence and theories of preferential choice. J Econ Lit. 2006; 44(3): 631-61. Publisher Full Text

40. Grisso T, Applebaum PS: Assessing Competence to Consent to Treatment. New York: Oxford University Press; 1998. Reference Source

41. Smith $\mathrm{RD}$, Coast $\mathrm{J}$ : Controlling antimicrobial resistance: a proposed transferable permit market. Health Policy. 1998; 43(3): 219-32. PubMed Abstract | Publisher Full Text

42. Culyer AJ: The Political Economy of Social Policy. Oxford: Martin Robertson 1983; 29-34. 


\section{Open Peer Review}

\section{Current Peer Review Status: ? $\checkmark$}

Version 1

Reviewer Report 07 March 2018

https://doi.org/10.5256/f1000research.14416.r30070

(C) 2018 Levin C. This is an open access peer review report distributed under the terms of the Creative Commons Attribution License, which permits unrestricted use, distribution, and reproduction in any medium, provided the original work is properly cited.

\section{Carol E. Levin}

Department of Global Health, University of Washington, Seattle, WA, USA

As a practitioner who conducts costs and cost-effectiveness analysis in low and middle-income countries, this was a useful paper and reminder to consider context and include stakeholder input early in the process as best practice. I appreciated the history of perspective and the clear conclusions simply stated. I have replied partly to two of reviewer questions because I read this opinion paper through the lens of a practitioner working only in a global health context, with familiarity of the published papers across a number of global health conditions.

As I read the paper, I wondered who the audience is for this paper? Is it researchers in highincome countries? Is it intended for researchers in global health economics? Is the purpose of the paper to counter recent recommendations of the second panel on cost-effectiveness or the second "Washington Panel"?

I wasn't quite sure what the problem being addressed is and who the intended audience for this paper was, since it combined a mix of practical points, theory and recommendations.

Recommendation: In the introduction, can the authors add a problem statement? And then explicitly indicate if these recommendations are for researchers in high-income countries or global health settings or both.

Also, as a practitioner, my experience is that analysts, conducting global health economic evaluations, more often than not, use common sense and already use a provider or (provider and societal perspective) in the majority of published economic evaluations in global health (whether for evaluating health technology, vaccines, diagnostics, medicines, delivery strategies). For instance, I selected two annexes available through the Disease Control Priorities Project for Reproductive Maternal and Child Health CEA studies and for Cardiovascular CEA studies and found that out of 295 articles, $22 \%$ were conducted from a societal perspective and $52 \%$ were from a provider perspective and 15\% were from both. For CVD prevention or treatment CEA studies, $27 \%$ were from a societal perspective and $68 \%$ from a provider perspective. 
This may not be the same as more deliberately using context specific perspective. So this either means that researchers conducting global health economic evaluations are already pragmatic, engaging stakeholders to select a context specific perspective, or that they are not following recommended guidelines. The authors note that only a third of the guidelines from both HIC and LMIC recommend a societal perspective. That certainly makes sense to me, for LMIC where economic evaluations are often conducted to introduce a new health technology or delivery strategy, where the status quo is nothing (or very low coverage of existing technologies or services).

As the authors nicely present, EE was method first developed for use in high-income countries, with a very specific objective in mind. They were not developed with global health interventions and strained governance and health systems in mind. However, as interest and capacity grows in LMIC to conduct EE, guidelines are extremely useful for researchers at nascent stages of learning a new method. And these guidelines typically get adapted initially and over time to better suit applications in global health, through disease specific recommendations/guidance/guidelines promoted by WHO or other multi-lateral or global health organizations.

On page 4, the authors note that there can be no presumption that one size fits all, and states we should not encourage a similar intensity to enter an 'authoritative methodological guide to best practice in EE'. I'm not sure that analysts DO presume a one size fits all. This is very clear from any review of a set of CEA studies that look at exactly the same two alternative strategies to address the same health condition. Comparability across many studies is difficult. I'm also not sure whom the authors are referring to and if there is a problem among researchers conducting global health EE with respect following authoritative guidelines (There are many problems in standard methods and reporting across studies-and these do cause a problem for using resulting study results-suggesting that guidelines are important to improve quality and comparability of studies-even when appearing authoritative-not sure what is wrong with that when talking about methods). When the guidance is not feasible or even does not make sense (i.e. using a societal perspective), it does seem that these recommendations are simply not followed. This may explain why provider perspective is used more often than provider perspective in EEs in LMIC.

Recommendation: Is it possible to take into consideration what is done in practice and acknowledge that practice already is very different from the guidance-certainly in LMIC?

Recommendation: On page 3, column 1, par 2, suggest indicating that guides to best practice can be traced back at least as far as 1980 developed for use in high income countries to inform government allocation of resources (or something to this effect). Very important to note these guidelines were NOT initially developed for LMIC, but adapted more recently for that purpose.

Quality and comparability in economic evaluations is challenging. In the introduction, the authors recognize the need to set international quality standards, but I take some issue in calling those that follow the standards falling into a "trap of small-town thinking". Perhaps this is not what the authors meant, but I'm not sure that it is parochialism at work, but rather a desire to generate comparable results, using economic theory as a foundation for the recommendation. Perhaps it is this underlying theory that the authors take issue with.

The authors understand the history and economic theory underlying the recommendation of a societal perspective much better than I, but it is unclear to me what the economic debate is and 
between who? They allocate a section in the paper to a theoretical discussion on the issue of individual expressions of value and collective ones. While I found this interesting, it seemed somewhat out of place to me, if this paper's audience is practitioners in low and middle-income countries, doing their best to figure out the best way to conduct economic evaluations.

Recommendation: What is the consequence of wrongly using a societal perspective? Can the authors more simply explain this to readers in the introduction or somewhere in the paper why a societal perspective may not allocate resource efficiently and equitably?

This gets me to my final comment on the paper, which it is a mix of a thought paper and a recommendation or guidance paper. I found myself wondering about the 'how' on page 9 when authors suggest engaging a long list of stakeholders in determining the perspective of the EE analysis. I agree with this, but can the authors refer the reader or add information on how to do this? This becomes particularly important for academics that are conducting EE in LMIC without direct access to stakeholders. This is often the case for researchers conducting EE for a specific health technology (vaccines, drugs, diagnostic, mhealth application,) that is under development. In addition, even when new services or technologies are funded and ready for introduction in LMIC, demand comes from the donor, researcher or NGO, not the in-country stakeholders that will ultimately introduce and fund the health intervention. However, I fully agree, when stakeholders are involved, identifying who will USE the information, and engaging them early makes it much easier to determine the objective and perspective of the study.

\section{Minor comment}

\section{Abstract}

1. Suggest that the authors provide a main recommendation in the abstract. Or end the abstract with a more positive statement stating that economic evaluation becomes more relevant when the perspective is sensitive to context and appropriate for the objective of the analysis.

Is the topic of the opinion article discussed accurately in the context of the current literature?

Partly

Are all factual statements correct and adequately supported by citations? Yes

Are arguments sufficiently supported by evidence from the published literature? Partly

Are the conclusions drawn balanced and justified on the basis of the presented arguments? Yes

Competing Interests: No competing interests were disclosed.

Reviewer Expertise: Global health economics

I confirm that I have read this submission and believe that I have an appropriate level of expertise to confirm that it is of an acceptable scientific standard, however I have 


\section{significant reservations, as outlined above.}

Author Response 21 Mar 2018

\section{Benjarin Santatiwongchai,}

The authors are grateful to the reviewers for the evident care they have taken in reviewing our paper.

Carol Levin requests a problem statement and greater clarity as to its intended readership. We thought we had stated the "problem" clearly in the Introduction, namely that what we called "small-town thinking" imposes arbitrary limits on the perspective from which economic evaluations are conducted. Furthermore, we do not regard it as the business of analysts (when acting as analysts) to assert appropriate perspectives. It follows that, since this is a point about scientific method, our article is addressed at one level to methodologists, textbook writers and others who seek to provide training materials and set standards of good practice for economic evaluations. Since good practice is something that practitioners ought to follow, practitioners need to be alerted to the possibility that not all expert recommendations on good practice may be sound. While this is a perfectly general point, which requires us all to be critically alert to the possibility of poor guidance, we quite specifically aimed a criticism at the common recommendation that studies be conducted from the "societal" perspective. We offered a little history of this approach, which has begun to assume the status of a common standard. The main reason for our view is that to select a perspective (any perspective) is to make a social value judgment about what "counts" in designing and implementing public policy, including health policy. The perspective defines the benefits, costs and harms that are to be considered as well as the ways in which they are to be brought into a single judgment about the eligibility of clinical procedures for inclusion in an insurance (public or private) scheme. Analysts are not empowered (qua analysts) to make these judgments. Those who are empowered include the managers of private health insurance schemes (typically accountable to a board, to trustees, or to shareholders) and the managers of public schemes, usually accountable to a minister and through the minister to an electorate. These people are commonly referred to as "decision makers". This accountability does not apply to analysts - unless of course they are themselves also managers, trustees, etc. Accordingly, good practice for analysts is to assist decision makers in selecting a perspective that is defensible and in accordance with the social and political values the decision makers in question are expected to live up to.

One way in which this can be done is to have the decision makers endorse a formal methodological approach that should characterise all the analysis commissioned for that specific jurisdiction. This is very common amongst jurisdictions that have set up specialist agencies to make decisions (like NICE) or to make recommendations (like HITAP or CADTH). We observed in our review that the societal perspective does not usually characterise these guides to method, or "reference cases". Nor are they by any means identical, despite having been devised with assistance of evidently competent economic analysts. This should not be a matter for regret. Rather, the presumption is that each has been designed for the particular circumstances of the jurisdiction to which it applies. Amongst other implications is that academics conducting analyses "without direct access to stakeholders", as Dr Levin puts it, are at great risk of producing recommendations that are irrelevant in any specific 
context. Good practice does not invariably require huge amounts of interaction with stakeholders but when the client for analysis has made no statement about perspective, and stakeholders are beyond reach, good practice is most likely to be "no practice". For whom, one might ask, might such an uninformed analysis be intended?

Competing Interests: None

Reviewer Report 24 January 2018

https://doi.org/10.5256/f1000research.14416.r29943

(C) 2018 Winch A. This is an open access peer review report distributed under the terms of the Creative Commons Attribution License, which permits unrestricted use, distribution, and reproduction in any medium, provided the original work is properly cited.

\section{Alexander Winch}

PRICELESS SA (Priority Cost Effective Lessons for System Strengthening South Africa), University of the Witwatersrand, Johannesburg, South Africa

\section{Is the topic of the opinion article discussed accurately in the context of the current literature?}

This is a welcome and much needed addition to the dialogue on improving the quality of conducting and reporting economic evaluations and HTA internationally.

Whilst the linkage is made well between the need for an explicit perspective as part of a robust high quality economic evaluation, one opportunity could be to explicitly state the importance of a well defined and robust decision problem as a fundamental to being able to define an appropriate perspective for an EE. Whilst this may be clear for those familiar with the guidelines, however the intended audience may not be familiar with the concept of scoped decision problems.

The section on the history of perspectives in methodological guidelines for conduction EE covers well the main evolution in guidelines. One potential enhancement could be through a discussion on why it took 20+ years for the perspective of an EE to be included explicitly as a characteristic in guidelines.

The article is clear on the justification for an explicit perspective, and why broader is not always better when it comes to 'societal perspective'. However, one opportunity for the article could be to look at the misuse or misunderstanding of concepts and language between policy makers and the technical EE analysts when it comes to the perspective on an evaluation.

For example, policy decision makers may be supportive of using a 'societal perspective' for an economic evaluation in a context for which this may not be appropriate, yet actually be conflating this concept of the 'societal perspective' with undertaking an inclusive, stakeholder engagement strategy in the health decision making process. 
This conflation, is similar to the use by policy decision makers of terms such as 'costeffective' of an intervention, even when this is not directly linked to the concept of opportunity cost, or the use of a context specific CE threshold, creating as a weird consequence the dichotomy of something being deemed 'cost-effective' yet 'unaffordable'.

\section{Are all factual statements correct and adequately supported by citations?}

All factual statements are correct and supported by citations.

Paragraph 7 could strengthen the assertion that 'Some LMICs, where out-of-pocket costs are significant select a societal perspective though very few LMICs have a track record of using EE systematically' through references to LMICs with high OOPs, or LMICs track record of EE use in decision making

Are arguments sufficiently supported by evidence from the published literature?

All arguments made in the article are supported by evidence from the published literature.

In paragraph 3, the assertion that 'a good study will be, therefore, explicit about its perspective' could be enhanced through using terms such as 'high quality', 'economically rigorous' or 'comprehensive' as this can then be referenced back to the principles of the iDSI reference case.

Are the conclusions drawn balanced and justified on the basis of the presented arguments? The conclusions drawn are balanced and justified on the basis of presented arguments.

Dependent on the intended audience for the article, the language could be simplified from 'context-free' and 'context-dependent' requirements, and instead restructure the conclusions for both the EE analyst (e.g. 1. advise, assist and point out likely consequences to decision maker 2) Not to engage in advocacy of any single perspective), and conclusions for the policy decision maker (1. adopt an explicit perspective in an EE 2. Be explicit in the reasons behind the choice of the perspective etc..)

\section{Minor additional comments:}

Table 2 displays payer, health care sector, societal as discrete categories - Does this double count those countries where multiple perspectives may be recommended in guidelines? Or are they given one discrete category? - For clarity it might be good to include a 'multiple' category to the table

For standardization and clarity, the language under column 'perspective' in Table 1 could be greater aligned. E.g:

'Costs: Health care payer (government + patients); outcomes: society'

'Health care sector....'

'In the reference case, the perspective should be that of the publicly funded health care payer.'

'Health technology assessment (HTA) in Indonesia is expected to use societal perspective.'

Last bullet point on conclusions is repeated

Is the topic of the opinion article discussed accurately in the context of the current 


\begin{abstract}
literature?
Yes

Are all factual statements correct and adequately supported by citations?

Yes
\end{abstract}

Are arguments sufficiently supported by evidence from the published literature?

Yes

Are the conclusions drawn balanced and justified on the basis of the presented arguments? Yes

Competing Interests: No competing interests were disclosed.

I confirm that I have read this submission and believe that I have an appropriate level of expertise to confirm that it is of an acceptable scientific standard.

Author Response 21 Mar 2018

Benjarin Santatiwongchai,

The authors have much sympathy with Alex Winch's comment on the ambiguity of language and the very real possibility that the understanding of decision makers and technical experts may be significantly divergent. This is probably something that can only be addressed through training (especially the training of health economists) and through careful explaining and re-explaining. It is not helped by devising new names for economic evaluation (currently running at around 24 near synonyms) or by inconsistent use of definitions even amongst economists (a spectacular demonstration of such disagreement about "opportunity cost" is Ferraro and Taylor 2005, but much the same embarrassing variation exists concerning "comparative and absolute advantage", "cost-effectiveness", "efficiency" or "equity").

Ferraro PJ, Taylor LO (2005) Do economists recognize an opportunity cost when they see one? A dismal performance from the dismal science, Contributions in Economic Analysis \& Policy, 4. doi:10.2202/1538-0645.1469.

Competing Interests: None

Reviewer Report 24 January 2018

https://doi.org/10.5256/f1000research.14416.r29941

(C) 2018 Tordrup D. This is an open access peer review report distributed under the terms of the Creative Commons Attribution License, which permits unrestricted use, distribution, and reproduction in any medium, provided the original work is properly cited. 


\author{
David Tordrup \\ 1 Triangulate Health Ltd, London, UK \\ 2 WHO Collaborating Centre for Pharmaceutical Policy and Regulation, University of Utrecht, \\ Utrecht, The Netherlands
}

The authors present a historical review of the origin of "perspective" in economic evaluation, and proceed to critique the standardisation of perspective, proposing that it is inherently contextspecific. The paper is interesting and well written. I would make the following points:

1. If we accept that guidelines for EE written by e.g. Drummond et al. are intended for evaluation of healthcare interventions, with the decision problem being to adopt or not adopt a new technology, and that these guidelines are at least implicitly developed with the UK context in mind, this means that - yes - the guidelines are intended to generalise away from a specific context (the technology) - but - they also generalise within a specific context (the health system). The same can be said for methods guidance published by NICE. So there are situations where the end users of evidence are issuing guidance on the approach to EE generation, to determine best practices and what evidence is acceptable to the decision problem. The authors concede this, I believe, when they write in conclusion "if the sponsors of a study intend it to inform policy decisions (public or otherwise) the decision makers' perspectives should be adopted (along with others deemed to be relevant by the sponsors)". This should mean that not all standardisation of perspective is necessarily bad.

2. Further to point 1, if the authors believe that each EE study intended to inform a public healthcare payer perspective should have its perspective built from scratch, how would the consumer of the evidence (the payer) avoid "cherry picking" by the sponsor (a manufacturer) of the most economically advantageous outcomes of the new technology under consideration? And if the perspective should not be built from scratch, how is that different from accepting to have some form of standardised guidance on perspective?

3. The authors also note capacity as a constraint in LMIC's, it seems point 2 would pose an even more substantial risk of "evidence capture" in such settings, where the majority of resource and technical capacity could easily be on the sponsor side, and not the evidence user side. In such cases, the capacity of the evidence user to articulate their needs could be severely limited.

So, while I fully agree that the choice of perspective should be determined by the user of the evidence, I think the authors should revisit their arguments against guidelines on perspective with the points above in mind. Are there cases where it is desirable to have standardised perspective guidance? What are the situations where it is undesirable - presumably where it is simply the result of asserting academic authority or other vested interest?

Is the topic of the opinion article discussed accurately in the context of the current literature?

Yes

Are all factual statements correct and adequately supported by citations?

Yes 
Are arguments sufficiently supported by evidence from the published literature? Yes

Are the conclusions drawn balanced and justified on the basis of the presented arguments? Partly

Competing Interests: No competing interests were disclosed.

I confirm that I have read this submission and believe that I have an appropriate level of expertise to confirm that it is of an acceptable scientific standard, however I have significant reservations, as outlined above.

Author Response 21 Mar 2018

Benjarin Santatiwongchai,

In response to David Tordrup, we should emphasise that we neither said nor implied that a "standard" perspective is "bad". The question is: who chooses the perspective? NICE, CADTH and HITAP each has a standard perspective that is fit for its local purpose and chosen by those accountable for making decisions or recommendations in that context. Each of the authors of this paper has been both analyst and decision maker (the latter as the employee or non-executive officer of an HTA agency\}. Our accountabilities in each role are not the same. In particular, our skills as scientists do not qualify us to make value judgments on behalf of society. At the most, the kind of value judgments we are entitled to make are value judgments about the quality of the science, which are certainly judgments of value, whether the science is good science, but they are not judgments about what is good for society. What qualified us as makers of social value judgments was our appointment through a legitimate process.

Our view on perspective does not involve every study building a perspective from scratch. On the contrary, there is much to be said for establishing agencies like NICE etc. which are competent to set standards appropriate to their local context. In many cases analysts might usefully be employed in helping to build such a standard "from scratch" but, once set up, the work becomes one of maintenance as new techniques of analysis emerge, or new data, or new criteria are suggested. As far as any specific study is concerned, its authors should follow the guidance as set out by decision makers or else guess as best they may what the perspective might be of those whose decisions they seek to influence.

Competing Interests: None 
The benefits of publishing with F1000Research:

- Your article is published within days, with no editorial bias

- You can publish traditional articles, null/negative results, case reports, data notes and more

- The peer review process is transparent and collaborative

- Your article is indexed in PubMed after passing peer review

- Dedicated customer support at every stage

For pre-submission enquiries, contact research@f1000.com 